\title{
Full counting statistics of a single-molecular quantum dot
}

\author{
Bing Dong, G. H. Ding, and X. L. Lei \\ Key Laboratory of Artificial Structures and Quantum Control (Ministry of Education), \\ Department of Physics, Shanghai Jiaotong University, 800 Dongchuan Road, Shanghai 200240, China
}

(Dated: November 17, 2018)

\begin{abstract}
We investigate the full counting statistics of a single quantum dot strongly coupled to a local phonon and weakly tunnel-connected to two metallic electrodes. By employing the generalized nonequilibrium Green function method and the Lang-Firsov transformation, we derive an explicit analytical formula for the cumulant generating function, which makes one to be able to identify distinctly the elastic and inelastic contributions to the current and zero-frequency shot noise. We find that at zero temperature, the inelastic effect causes upward steps in the current and downward jumps in the noise at the bias voltages corresponding to the opening of the inelastic channels, which are ascribed to the vibration-induced complex dependences of electronic self-energies on the energy and bias voltage. More interestingly, the Fano factor exhibits oscillatory behavior with increasing bias voltage and its minimum value is observed to be smaller than one half.

PACS numbers: 85.65.+h, 71.38.-k, 72.70.+m, 73.23.-b, 73.63.Kv
\end{abstract}

\section{INTRODUCTION}

Recent progress in nanotechnology has facilitated the fabrication of single-electron tunneling devices using organic molecules. A variety of intriguing effects has been observed in the transport properties of the single-molecule transistors (SMTs) due to the couplings between the intrinsic mechanical degree of freedom (phonon, vibron) in molecules and electrons during tunneling. $\stackrel{1-8}{-\underline{8}}$ For instance, the obvious phonon-assisted current steps have been measured in the current-biasvoltage characteristic of a variety of individual molecules connected to metal electrodes $\underline{\underline{1}} \underline{\underline{-6}}$ More interesting transport properties, e.g. the Franck-Condon blockade in the current steps and negative differential conductance due to nonequilibrated phonon excitation, have been experimentally demonstrated in the device of a suspended single-wall carbon nanotube $\stackrel{7,8}{\underline{7}}$

These experimental observations have stimulated great interest in the theoretical investigations. In recent years, a variety of different approaches have been developed to study the transport properties and current fluctuation characteristics in the electron-phonon coupled systems, mainly containing the kinetic-equation approach (master equations),,$\frac{9-18}{9}$ the nonequilibrium Green function (NGF) techniques, $\frac{19}{2}-37$ and the diagrammatic Monte Carlo simulation ${ }^{38}$ It is well-known that the NGF is a most powerful method to study nonequilibrium behavior of a many-body system. Within the NGF theoretical formulation, various self-consistent second-order perturbation calculations have been carried out, on the weak electron-phonon interaction (EPI) strength, to examine the inelastic correction to the nonlinear conductance of the SMTs $\underline{19}-25$ On the other hand, in order to study the strong EPI effects, two authors of this paper proposed a nonperturbative analysis of the inelastic effects on current ${ }^{26-30}$ and its fluctuations ${ }^{30}$ by mapping of the many-body EPI problem onto a multichannel singleelectron scattering problem. ${ }^{39}$ This mapping technique is only valid in the limit of weak electronic tunnel-couplings between the molecular quantum dot (QD) and electrodes since the so-called Fermi sea effect is neglected in the mapping procedure. To circumvent this drawback and to cover more wide ranges of system parameters, e.g. the EPI and tunnel-coupling strengths, another nonperturbative scheme has been recently developed based on the NGF in the polaron representation $=\underline{31}-34,36,37$ In particular, Galperin et.al. formulated a fully self-consistent solution of both electronic and phononic GFs by employing equation of motion method to establish the Dyson-type coupled equations. ${ }^{34}$ Later on, he further developed this approach to study the zero-frequency noise spectrum of SMTs $\stackrel{35}{3}$ Because the Wick theorem can not be applied to calculate the current-current correlation function of the EPI system, he instead made use of the noise formula of the noninteracting system and simply replaced the electronic GFs in the noninteracting noise formula with the self-consistently calculated ones. Moreover, this approach was extended to consider the inelastic effect of multimode vibrational dynamics, 36

Nowadays, there is continually increasing interest in the full counting statistics (FCS) of charge transport in nanocale system. ${ }^{40.41}$ This remarkable concept was first proposed by Levitov and Lesovik to describe the whole probability distribution of transmitted charge during a fixed time interval in a mesoscopic conductor $\stackrel{42}{\underline{4} \text { It is }}$ therefore an intriguing task to examine the FCS of electronic tunneling in the presence of EPI. Employing the master equations, the inelastic effect on the FCS has been studied in the resonant tunneling regime $\stackrel{13,17,18}{\text { For the }}$ phase-coherent transport through an interacting system, the NGF is required. Recently, Gogolin and Komnik have generalized the Meir-Wingreen NGF formulation for the quantum transport in mesoscopic system to the FCS issue, and derived a generic expression for the generating function of the cumulants expressed only in terms of the local Keldysh GFs of the central region, which is valid in any types of the central region, noninteract- 
ing or interacting. ${ }^{43}$ In this theory, it is no need to directly calculate the current-current correlation functions by employing the Feynman diagram technique. Instead a Schwinger external source, i.e. here a fictitious measuring field $\lambda$ in the tunneling Hamiltonian, is introduced to count the numbers of transmitted electrons and functional derivative is provoked at the end of calculation to generate the cumulants of charge current distribution. 44 Another advantage of this Hamiltonian approach is that it can automatically contain the vertex corrections in the current-current correlation functions. With help of the generalized Schwinger-Keldysh GF technique, inelastic effects on the FCS in SMTs have been recently investigated, in which a compact analytic expression for the FCS was derived under the assumption that vibration mode is at equilibriated state. ${ }^{45}$ These authors focused their studies on the concrete behaviors of the current and shot noise jumps, upward or downward, due to phonon excitation when the first inelastic channel is opening. Remarkably, the negative contribution to noise due to vibration excitation has been experimentally observed by recent shot noise measurements on $\mathrm{Au}$ atomic contact, 46 and has been further confirmed by a subsequent calculation of the inelastic shot noise signals in $\mathrm{Au}$ and $\mathrm{Pt}$ atomic point contacts from first principles $\stackrel{47}{4}$ Moreover, the effect of vibrational heating on FCS has been further considered,,$\frac{48,49}{4}$ and analytical results on FCS accounting for nonequilibrium phonon distributions have been obtained ${ }^{50}$ Nevertheless, all these studies employed the second-order perturbation expansion to evaluate the electronic and phononic self-energies, and consequently they are valid for the regime of weak EPI. The full knowledge of the inelastic effects on FCS in the regime of strong EPI is still less studied. 51 This constitutes the purpose of the present paper.

The rest of the paper is organized as follows. In Sec. II, we introduce the model Hamiltonian of a molecular QD. In Sec. III, we present the theoretical formulation for the FCS calculation in the presence of EPI. In particular, the explicit expressions of the FCS, current, and zerofrequency shot noise are derived. In Sec. IV, we carry out numerical calculations of differential conductance, shot noise and Fano factor, and discuss these results. Finally, a brief summary is given in Sec. V.

\section{MODEL}

In this paper, we consider a simple model for a molecular QD with one spinless level (electronic energy $\epsilon_{d}$ ) coupled to two electrodes left $(\mathrm{L})$ and right $(\mathrm{R})$ (each a freee electron reservoir at its own equilibrium), and also linearly coupled to a single vibrational mode (phonon) of the molecule having frequency $\omega_{0}$ with coupling strength $g_{e p}$. The model Hamiltonian is

$$
H=H_{l e a d s}+H_{m o l}+H_{T}
$$

with

$$
\begin{aligned}
H_{\text {leads }} & =\sum_{\eta, \mathbf{k}} \varepsilon_{\eta \mathbf{k}} c_{\eta \mathbf{k}}^{\dagger} c_{\eta \mathbf{k}}, \\
H_{m o l} & =\varepsilon_{d} d^{\dagger} d+\omega_{0} a^{\dagger} a+g_{e p} d^{\dagger} d\left(a^{\dagger}+a\right), \\
H_{T} & =\sum_{\eta, \mathbf{k}}\left(\gamma_{\eta} e^{-i \lambda_{\eta}(t) / 2} c_{\eta \mathbf{k}}^{\dagger} d+\text { H.c. }\right)
\end{aligned}
$$

where $c_{\eta \mathbf{k}}^{\dagger}\left(c_{\eta \mathbf{k}}\right)$ is the creation (annihilation) operator of an electron with momentum $\mathbf{k}$, and energy $\varepsilon_{\eta \mathbf{k}}$ in lead $\eta(\eta=L, R)$, and $d^{\dagger}(d)$ is the corresponding operator for a spinless electron in the QD. $a^{\dagger}(a)$ is phonon creation (annihilation) operators for the vibrational mode (energy quanta $\left.\omega_{0}\right) . \quad \gamma_{\eta}$ describes the tunnel-coupling matrix element between the QD and lead $\eta$. The corresponding coupling strength is defined as $\Gamma_{\eta}=2 \pi \sum_{k}\left|\gamma_{\eta}\right|^{2} \delta\left(\omega-\varepsilon_{\eta \mathbf{k}}\right)$, which is assumed to be independent of energy in the wide band limit. In order to investigate the full counting statistics (FCS), an artificially measuring field $\lambda_{\eta}(t)$ is introduced with respect to the lead $\eta$ on the Keldysh contour: $\lambda_{\eta}(t)=\lambda_{\eta-} \theta(t) \theta(\mathcal{T}-t)$ on the forward path and $\lambda_{\eta}(t)=\lambda_{\eta+} \theta(t) \theta(\mathcal{T}-t)$ on the backward path $(\mathcal{T}$ is the measuring time during which the counting fields are non-zero and the counting fields will be set to be opposite constants on the forward and backward Keldysh contour as $\lambda_{\eta-}=-\lambda_{\eta+}=\lambda_{\eta}$ in the final derivation) 43,44 Throughout we will use natural units $e=\hbar=k_{\mathrm{B}}=1$.

For dealing with the problem involving strong electronphonon interaction, it is very convenient to apply a standard Lang-Firsov canonical transformation, $S=$ $g d^{\dagger} d\left(a^{\dagger}-a\right)\left(g=g_{e p} / \omega_{0}\right)$, to the Hamiltonian Eq. (1a), 52 leading to a transformed Hamiltonian

$$
\begin{aligned}
\widetilde{H} & =e^{S} H e^{-S}=H_{\text {leads }}+\widetilde{H}_{m o l}+\widetilde{H}_{T}, \\
\widetilde{H}_{m o l} & =\widetilde{\varepsilon}_{d} \widetilde{d}^{\dagger} \widetilde{d}+\omega_{0} a^{\dagger} a=\widetilde{\varepsilon}_{d} d^{\dagger} d+\omega_{0} a^{\dagger} a, \\
\widetilde{H}_{T} & =\sum_{\eta, \mathbf{k}}\left(\gamma_{\eta} e^{-i \lambda_{\eta}(t) / 2} c_{\eta \mathbf{k}}^{\dagger} \widetilde{d}+\text { H.c. }\right) \\
& =\sum_{\eta, \mathbf{k}}\left(\gamma_{\eta} e^{-i \lambda_{\eta}(t) / 2} c_{\eta \mathbf{k}}^{\dagger} d X+\text { H.c. }\right) .
\end{aligned}
$$

Here $\widetilde{\varepsilon}_{d}=\varepsilon_{d}-\frac{g_{e p}^{2}}{\omega_{0}}$ is the renormalized energy level of the $\mathrm{QD}$ and $\widetilde{d}=d X$ denotes the new Fermionic operator dressed by the phononic shift operator $X$,

$$
X=e^{g\left(a-a^{\dagger}\right)} .
$$

Therefore, the transformed Hamiltonian is equivalent to a noninteracting resonant-level model with a vibration modified dot-lead tunneling described by the shift operator $X$ in Eq. (2c), which is responsible for the observation of the Franck-Condon steps in the current-voltage characteristics of the single molecular transistor. This noninteracting effective Hamiltonian $\widetilde{H}$ Eq. (2a) is our starting point for the FCS investigation in the following section. 


\section{THEORETICAL METHODS}

\section{A. Adiabatic Potential for FCS}

To investigation the probability distribution $P_{q_{L}, q_{R}}$ of the charge $q_{\eta}$ to be transferred through the QD to lead $\eta$ during the measuring time, we should calculate the so-called cumulant generating function (CGF) $\chi(\lambda) \equiv \chi\left(\lambda_{L}, \lambda_{R}\right)=\sum_{q_{L}, q_{R}} P_{q_{L}, q_{R}} e^{i \sum_{\eta} q_{\eta} \lambda_{\eta}}$ for the twoterminal QD, which can be determined as a Keldysh partition function: 44

$$
\chi(\lambda)=\left\langle T_{\mathcal{C}} e^{-i \int_{\mathcal{C}} \widetilde{H}_{T}(t) d t}\right\rangle_{\lambda},
$$

where $T_{\mathcal{C}}$ denotes time ordering along the SchwingerKeldysh contour $\mathcal{C}$ and the expectation value is written in the interaction picture with respect to the effective Hamiltonian, $H_{\text {leads }}+\widetilde{H}_{m o l}$. According to Ref. 43, to calculate the CGF $\chi(\lambda)$ it is technically more convenient employing the adiabatic potential method: $\ln \chi(\lambda)=$ $-i \mathcal{T U}\left(\lambda_{-}, \lambda_{+}\right)=-i \mathcal{T} \mathcal{U}(\lambda,-\lambda)$, where the adiabatic potential $\mathcal{U}\left(\lambda_{-}, \lambda_{+}\right)$is defined due to the nonequilibrium Feynman-Hellmann theorem as

$$
\begin{aligned}
\frac{\partial \mathcal{U}\left(\lambda_{-}, \lambda_{+}\right)}{\partial \lambda_{\eta-}} & =\left\langle\frac{\partial \widetilde{H}_{T}(t)}{\partial \lambda_{\eta-}}\right\rangle_{\lambda} \\
& =-\frac{i}{2} \sum_{\mathbf{k}}\left\langle\gamma_{\eta} e^{-i \lambda_{\eta-} / 2} c_{\eta \mathbf{k}}^{\dagger} \tilde{d}-\text { H.c. }\right\rangle_{\lambda}
\end{aligned}
$$

with the notation

$$
\langle\cdots\rangle_{\lambda}=\frac{1}{\chi\left(\lambda_{-}, \lambda_{+}\right)}\left\langle T_{\mathcal{C}} \cdots e^{-i \int_{\mathcal{C}} \widetilde{H}_{T}(t) d t}\right\rangle_{0} .
$$

The further evaluation of the adiabatic potential amounts to calculations of the mixed GFs, $G_{d \eta \mathbf{k}}\left(t, t^{\prime}\right)=$ $-i\left\langle T_{\mathcal{C}} \widetilde{d}(t) c_{\eta \mathbf{k}}^{\dagger}\left(t^{\prime}\right)\right\rangle_{\lambda}$ and $G_{\eta \mathbf{k} d}\left(t, t^{\prime}\right)=-i\left\langle T_{\mathcal{C}} c_{\eta \mathbf{k}}(t) \widetilde{d}^{\dagger}\left(t^{\prime}\right)\right\rangle_{\lambda}$, as $\left(t^{+}=t+0^{+}\right)$

$$
\begin{aligned}
\frac{\partial \mathcal{U}\left(\lambda_{-}, \lambda_{+}\right)}{\partial \lambda_{\eta-}}= & \frac{\gamma_{\eta}}{2} \sum_{\mathbf{k}}\left[e^{-i \lambda_{\eta-} / 2} G_{d \eta \mathbf{k}}^{--}\left(t, t^{+}\right)\right. \\
& \left.-e^{i \lambda_{\eta-} / 2} G_{\eta \mathbf{k} d}^{--}\left(t, t^{+}\right)\right]
\end{aligned}
$$

Bearing in mind the facts that the transformed Hamiltonian is noninteracting one and the canonical transformation do not alter the canonical commutation relations between Fermionic operators, these mixed GFs can be cast into combinations of the contour-ordered GFs of the QD involving dressed electronic operators, $G_{d}\left(t, t^{\prime}\right)$, and bare lead GFs, $g_{\eta \mathbf{k}}\left(t, t^{\prime}\right)$,

$$
\begin{aligned}
G_{d \eta \mathbf{k}}\left(t, t^{\prime}\right) & =\int_{\mathcal{C}} d t^{\prime \prime} G_{d}\left(t, t^{\prime \prime}\right) \gamma_{\eta} e^{i \lambda_{\eta}\left(t^{\prime \prime}\right) / 2} g_{\eta \mathbf{k}}\left(t^{\prime \prime}, t^{\prime}\right) \\
G_{\eta \mathbf{k} d}\left(t, t^{\prime}\right) & =\int_{\mathcal{C}} d t^{\prime \prime} g_{\eta \mathbf{k}}\left(t, t^{\prime \prime}\right) \gamma_{\eta} e^{-i \lambda_{\eta}\left(t^{\prime \prime}\right) / 2} G_{d}\left(t^{\prime \prime}, t^{\prime}\right),
\end{aligned}
$$

with

$$
G_{d}\left(t, t^{\prime}\right)=-i\left\langle T_{\mathcal{C}} \widetilde{d}(t) \widetilde{d}^{\dagger}\left(t^{\prime}\right)\right\rangle_{\lambda}
$$

$$
\begin{aligned}
& =-i\left\langle T_{\mathcal{C}} d(t) X(t) X^{\dagger}\left(t^{\prime}\right) d^{\dagger}\left(t^{\prime}\right)\right\rangle_{\lambda} \\
g_{\eta \mathbf{k}}\left(t, t^{\prime}\right) & =-i\left\langle T_{\mathcal{C}} c_{\eta \mathbf{k}}(t) c_{\eta \mathbf{k}}^{\dagger}\left(t^{\prime}\right)\right\rangle_{\lambda} .
\end{aligned}
$$

Performing the Keldysh disentanglement and substituting the results back into Eq. (6) one obtains

$$
\begin{aligned}
\frac{\partial \mathcal{U}\left(\lambda_{-}, \lambda_{+}\right)}{\partial \lambda_{\eta-}}= & \sum_{\mathbf{k}} \frac{\gamma_{\eta}^{2}}{2} \int d t_{1}\left[e^{-i \bar{\lambda}_{\eta} / 2} G_{d}^{-+}\left(t, t_{1}\right) g_{\eta \mathbf{k}}^{+-}\left(t_{1}, t^{+}\right)\right. \\
& \left.-e^{i \bar{\lambda}_{\eta} / 2} g_{\eta \mathbf{k}}^{-+}\left(t, t_{1}\right) G_{d}^{+-}\left(t_{1}, t^{+}\right)\right]
\end{aligned}
$$

with $\bar{\lambda}_{\eta}=\lambda_{\eta-}-\lambda_{\eta+}$. It is noticed that the adiabatic potential Eq. (9) is exactly equivalent to that given by Maier in Ref. 51. Until now, all derivations are exact and what is done next is to calculate the dressed electronic $\mathrm{GF} G_{d}^{\alpha \beta}\left(t, t^{\prime}\right)(\alpha, \beta=+,-)$.

\section{B. Nonequilibrium GF approach for electron-phonon coupled system}

Following Galperin et al.,$\frac{34}{3}$ we can use the usual BornOppenheimer adiabatic approximation to decouple electron and phonon dynamics, which leads to a factorized form of the $\mathrm{GF} G_{d}\left(t, t^{\prime}\right)$ as a product of a pure electronic part and a phononic part,, 32

$$
G_{d}^{\alpha \beta}\left(t, t^{\prime}\right) \approx G_{c}^{\alpha \beta}\left(t, t^{\prime}\right) K^{\alpha \beta}\left(t, t^{\prime}\right)
$$

where

$$
\begin{aligned}
G_{C}\left(t, t^{\prime}\right) & =-i\left\langle T_{\mathcal{C}} d(t) d^{\dagger}\left(t^{\prime}\right)\right\rangle_{\lambda}, \\
K\left(t, t^{\prime}\right) & =\left\langle T_{\mathcal{C}} X(t) X^{\dagger}\left(t^{\prime}\right)\right\rangle_{\lambda} .
\end{aligned}
$$

The corresponding Feynman diagram in perturbation theory is shown schematically in Fig. 1(a). This decoupling is valid in the limit of a weak molecule-lead tunnelcouplings implying a relatively long residence time of the electron on the molecule, i.e., $\Gamma_{\eta} \ll \omega_{0}$.

Furthermore, we assume a extremely strong dissipation of the primary phonon mode to a thermal bath, e.g., to a substrate or a backgate. This means that the oscillator restores to its equilibrium state so quickly that it has no time to play a reaction to the electronic system when it is stimulated to an unequilibrated state by externalbias-voltage-driven tunneling electrons. In this situation, the oscillator can be described by an equilibrium Bose distribution $n_{B}=\left(e^{\omega_{0} / T}-1\right)^{-1}$ at the temperature $T$ and the phonon shift generator GF $K\left(t, t^{\prime}\right)$ can be replaced by its equilibrium correlation function,, 52

$$
K\left(t, t^{\prime}\right)=\left(\begin{array}{cc}
e^{-\phi(|\tau|)} & e^{-\phi(\tau)} \\
e^{-\phi(-\tau)} & e^{-\phi(-|\tau|)}
\end{array}\right)
$$

where $\phi(\tau)$ is defined as $\left(\tau=t-t^{\prime}\right)$

$$
\phi(\tau)=g^{2}\left[n_{B}\left(1-e^{i \omega_{0} \tau}\right)+\left(n_{B}+1\right)\left(1-e^{-i \omega_{0} \tau}\right)\right] .
$$


(a)

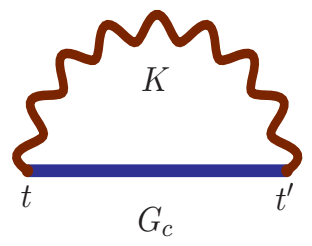

(b)

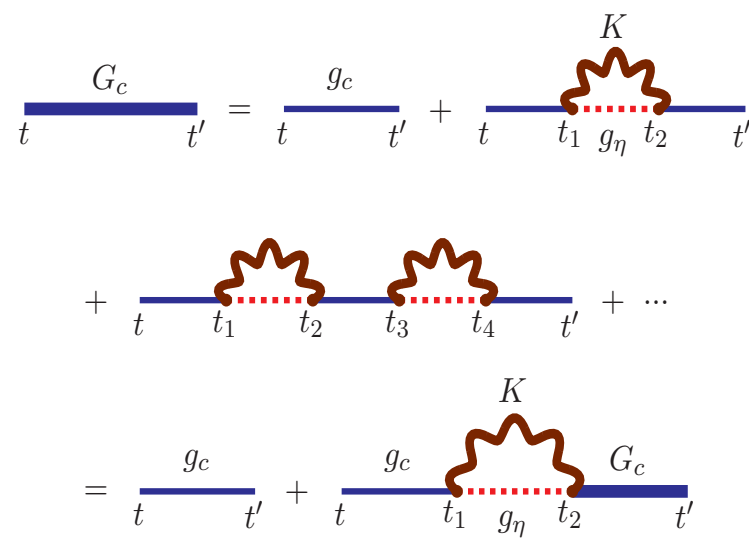

Figure 1: (Colour online) The Feynman diagrams taken into account in our calculations for the EPI system. (a) The diagram for the factorized GF $G_{d}\left(t, t^{\prime}\right)$. The thick solid line denotes the pure electronic GF $G_{c}\left(t, t^{\prime}\right)$ and the wiggly line represents the phonon cloud propagator $K\left(t, t^{\prime}\right)$. (b) The set of Feynman diagrams and Dyson equation for the pure electronic GF $G_{c}\left(t, t^{\prime}\right)$. The thin solid line is the bare dot GF $g_{c}$, and the dashed-line denotes the GF $g_{\eta}(\eta=L, R)$ of the leads.

It is noted that in this approximation, the phononic GF $K\left(t, t^{\prime}\right)$ becomes irrespective of the counting field $\lambda$.

Therefore, the next step is to calculate the contourordered electronic GF of the QD, $G_{c}^{\alpha \beta}\left(t, t^{\prime}\right)$, based on the transformed Hamiltonian $\widetilde{H}$ Eq. (2a). Nevertheless, since the transformed tunneling Hamiltonian Eq. (2C) involves the exponential operators $X$ and $X^{\dagger}$, one can not apply Wick's theorem to derive the Dyson-like equation of the pure electronic GF $G_{c}$. As an alternative method, an equation-of-motion (EOM) procedure has been usually used as approximations in literature ${ }^{32,34,53}$ Here, using the transformed Hamiltonian $\widetilde{H}$, we derive the EOM for the contour-ordered electronic GF $G_{c}\left(t, t^{\prime}\right)$ as

$$
\begin{aligned}
& \left(i \frac{\partial}{\partial t}-\tilde{\varepsilon}_{d}\right) G_{c}\left(t, t^{\prime}\right)=\delta_{\mathcal{C}}\left(t-t^{\prime}\right) \\
& -\sum_{\eta \mathbf{k}} i \gamma_{\eta} e^{i \lambda_{\eta}(t) / 2}\left\langle T_{\mathcal{C}} X^{\dagger}(t) c_{\eta \mathbf{k}}(t) d^{\dagger}\left(t^{\prime}\right)\right\rangle_{\lambda} .
\end{aligned}
$$

Then we consider the EOM for the three-point GF $\left\langle T_{\mathcal{C}} X^{\dagger}(t) c_{\eta \mathbf{k}}\left(t_{1}\right) d^{\dagger}\left(t^{\prime}\right)\right\rangle_{\lambda}$. It gives

$$
\begin{aligned}
& \left(i \frac{\partial}{\partial t_{1}}-\tilde{\varepsilon}_{\eta \mathbf{k}}\right)\left\langle T_{\mathcal{C}} X^{\dagger}(t) c_{\eta \mathbf{k}}\left(t_{1}\right) d^{\dagger}\left(t^{\prime}\right)\right\rangle_{\lambda} \\
& =\gamma_{\eta} e^{-i \lambda_{\eta}\left(t_{1}\right) / 2}\left\langle T_{\mathcal{C}} X^{\dagger}(t) X\left(t_{1}\right) d\left(t_{1}\right) d^{\dagger}\left(t^{\prime}\right)\right\rangle_{\lambda},
\end{aligned}
$$

or in the integration form as

$$
\begin{aligned}
& \left\langle T_{\mathcal{C}} X^{\dagger}(t) c_{\eta \mathbf{k}}\left(t_{1}\right) d^{\dagger}\left(t^{\prime}\right)\right\rangle_{\lambda}=\gamma_{\eta} \int_{C} d t_{2} e^{-i \lambda_{\eta}\left(t_{2}\right) / 2} \\
& \times g_{\eta \mathbf{k}}\left(t_{1}, t_{2}\right)\left\langle T_{\mathcal{C}} X^{\dagger}(t) X\left(t_{2}\right) d\left(t_{2}\right) d^{\dagger}\left(t^{\prime}\right)\right\rangle_{\lambda} .
\end{aligned}
$$

By taking the time limit $t_{1} \rightarrow t$ in the above equation, and substitute it to Eq. (15), one can obtain the EOM for $\mathrm{GF} G_{c}$ exactly as follows

$$
\begin{aligned}
& \left(i \frac{\partial}{\partial t}-\tilde{\varepsilon}_{d}\right) G_{c}\left(t, t^{\prime}\right)=\delta_{\mathcal{C}}\left(t-t^{\prime}\right)-\sum_{\eta \mathbf{k}} \int_{C} d t_{1} i \gamma_{\eta}^{2} \\
& \times e^{i\left[\lambda_{\eta}(t)-\lambda_{\eta}\left(t_{1}\right)\right] / 2} g_{\eta \mathbf{k}}\left(t, t_{1}\right)\left\langle T_{\mathcal{C}} X^{\dagger}(t) X\left(t_{1}\right) d\left(t_{1}\right) d^{\dagger}\left(t^{\prime}\right)\right\rangle_{\lambda} .
\end{aligned}
$$

Then we will make an approximation the same as in Eq. (10) to decompose the dressed propagator:

$$
\left\langle T_{\mathcal{C}} X^{\dagger}(t) X\left(t_{1}\right) d\left(t_{1}\right) d^{\dagger}\left(t^{\prime}\right)\right\rangle_{\lambda} \approx i K\left(t_{1}, t\right) G_{c}\left(t_{1}, t^{\prime}\right),
$$

and consequently obtain the Dyson equation for $G_{c}$

$$
\left(i \frac{\partial}{\partial t}-\tilde{\varepsilon}_{d}\right) G_{c}\left(t, t^{\prime}\right)=\delta_{\mathcal{C}}\left(t-t^{\prime}\right)+\int_{C} \Sigma_{c \lambda}\left(t, t_{1}\right) G_{c}\left(t_{1}, t^{\prime}\right),
$$

in which $\Sigma_{c \lambda}\left(t, t_{1}\right)$ is the contour-ordered electronic selfenergies in the time domain, which includes all couplings of the electronic degrees of freedom on the $\mathrm{QD}$ with those in the electrodes and the vibrational mode, and the counting fields as well,

$$
\Sigma_{c \lambda}^{\alpha \beta}\left(t, t_{1}\right)=\sum_{\eta \mathbf{k}} e^{i\left(\lambda_{\eta \alpha}-\lambda_{\eta \beta}\right) / 2} \gamma_{\eta}^{2} g_{\eta \mathbf{k}}^{\alpha \beta}\left(t, t_{1}\right) K^{\beta \alpha}\left(t_{1}, t\right) .
$$

The Dyson equation can also be written as an integration in terms of the pure electronic GF $G_{c}$,

$G_{c}\left(t, t^{\prime}\right)=g_{c}\left(t, t^{\prime}\right)+\int_{\mathcal{C}} d t_{1} d t_{2} g_{c}\left(t, t_{1}\right) \Sigma_{c \lambda}\left(t_{1}, t_{2}\right) G_{c}\left(t_{2}, t^{\prime}\right)$.

where $g_{c}\left(t, t^{\prime}\right)$ denotes the free electron GF for the dot without tunneling-coupling. It is clear that the ensuring GF $G_{c}\left(t, t^{\prime}\right)$ corresponds to summing over all the diagrams as shown in Fig. 1(b). This means that the present method accounts the vibration-modified-effect on electronic tunneling processes by embedding the phononic propagator into the tunneling self-energies. While the polaron tunneling approximation (PTA) scheme developed in Ref. 51 considers the vibrational effect only in the bare electronic GF, $g_{c}$, but remains the tunneling self-energies unmodified by phonon cloud (see the corresponding Feynman diagram, Fig. 3 in Ref. 51). On the other hand, our Dyson series for $G_{c}$ is also different from those of single particle approximation, $\stackrel{31,32}{\underline{G} \text { wich }}$ performs the same factorization for the full GF $G_{d}$ as ours but take no account of the phonon cloud in the Dyson series for $G_{c}$.

Now we accomplish our calculation for the pure electronic GF $G_{c}$. Projecting Eq. (22) onto the real time axis 
according to Langreth analytical continuation rules, and then performing Fourier transformation of the resulting equations gives an explicit expression for the electronic
GF $G_{c}(\omega)$ (Noting that the counting fields $\lambda_{\eta}(t)$ are taken to be opposite constants in time on the forward and backward Keldysh contour):

$$
G_{c}(\omega)=\frac{1}{\mathcal{D}_{\lambda}(\omega)}\left(\begin{array}{cc}
\omega-\tilde{\epsilon}_{d}+\Sigma_{c 0}^{+-}(\omega)-\Sigma_{c}^{r}(\omega) & \Sigma_{c \lambda}^{-+}(\omega) \\
\Sigma_{c \lambda}^{+-}(\omega) & -\left[\omega-\tilde{\epsilon}_{d}-\Sigma_{c 0}^{-+}(\omega)-\Sigma_{c}^{r}(\omega)\right]
\end{array}\right)
$$

with

$$
\begin{aligned}
\mathcal{D}_{\lambda}(\omega)= & {\left[\omega-\widetilde{\epsilon}_{d}-\Sigma_{c}^{r}(\omega)\right]\left[\omega-\tilde{\epsilon}_{d}-\Sigma_{c}^{a}(\omega)\right]+\Gamma_{L} \Gamma_{R} \sum_{n m} w_{n} w_{m}\left\{f_{L}\left(\omega+n \omega_{0}\right)\left[1-f_{R}\left(\omega-m \omega_{0}\right)\right]\left[e^{i\left(\bar{\lambda}_{L}-\bar{\lambda}_{R}\right) / 2}-1\right]\right.} \\
& \left.+f_{R}\left(\omega+n \omega_{0}\right)\left[1-f_{L}\left(\omega-m \omega_{0}\right)\right]\left[e^{-i\left(\bar{\lambda}_{L}-\bar{\lambda}_{R}\right) / 2}-1\right]\right\}
\end{aligned}
$$

where the lesser and greater self-energies of the electron can be expressed in frequency domain as

$$
\begin{aligned}
& \Sigma_{c \lambda}^{-+}(\omega)=\sum_{n=-\infty}^{\infty} w_{n} \Sigma_{c \lambda}^{(0),-+}\left(\omega+n \omega_{0}\right), \\
& \Sigma_{c \lambda}^{+-}(\omega)=\sum_{n=-\infty}^{\infty} w_{n} \Sigma_{c \lambda}^{(0),+-}\left(\omega-n \omega_{0}\right), \\
& \Sigma_{c 0}^{ \pm \mp}(\omega)=\left.\Sigma_{c \lambda}^{ \pm \mp}(\omega)\right|_{\lambda=0},
\end{aligned}
$$

and

$$
\begin{aligned}
& \Sigma_{c \lambda}^{(0),-+}(\omega)=i \sum_{\eta} e^{i \bar{\lambda}_{\eta} / 2} \Gamma_{\eta} f_{\eta}(\omega), \\
& \Sigma_{c \lambda}^{(0),+-}(\omega)=-i \sum_{\eta} e^{-i \bar{\lambda}_{\eta} / 2} \Gamma_{\eta}\left[1-f_{\eta}(\omega)\right] .
\end{aligned}
$$

Here $f_{\eta}=\left[1+\exp \left(\omega-\mu_{\eta}\right) / T\right]^{-1}$ is the Fermi distribution function at temperature $T$ and chemical potential $\mu_{\eta}=E_{F}+V_{\eta}$ of lead $\eta\left(E_{F}\right.$ is the Fermi energy and $V_{\eta}$ is the bias-voltage applied to lead $\eta$ ). The factor $w_{n}$ is the weighting factor describing the electronic tunneling involving absorption or emission of $n$ phonons. At a finite temperature,

$$
w_{n}=e^{-g^{2}\left(2 N_{B}+1\right)} e^{n \omega_{0} / 2 T} I_{n}\left(2 g^{2} \sqrt{n_{B}\left(n_{B}+1\right)}\right),
$$

where $I_{n}(x)$ is the $n$th Bessel function of complex argument. Moreover, the retarded self-energy in time domain can be defined in the usual way from the lesser and greater counterparts, $\Sigma_{c}^{r}(\tau)=\theta(\tau)\left[\Sigma_{c 0}^{+-}(\tau)-\Sigma_{c 0}^{-+}(\tau)\right]$, and thus its expression in frequency domain is

$$
\begin{aligned}
\Sigma_{c}^{r}(\omega)= & \sum_{\eta n} w_{n} \int \frac{d \omega^{\prime}}{2 \pi}\left\{\frac{\Gamma_{\eta} f_{\eta}\left(\omega^{\prime}\right)}{\omega+n \omega_{0}-\omega^{\prime}+i 0^{+}}\right. \\
& \left.+\frac{\Gamma_{\eta}\left[1-f_{\eta}\left(\omega^{\prime}\right)\right]}{\omega-n \omega_{0}-\omega^{\prime}+i 0^{+}}\right\} .
\end{aligned}
$$

It is observed that the vibration-modified electronic selfenergy due to tunneling is highly dependent on the applied bias voltage as shown in Fig. 2 in the following section, in contrast to the noninteracting QD-lead system where the tunneling induced self-energy is assumed to be a constant, $\Sigma^{r}(\omega)=-i\left(\Gamma_{L}+\Gamma_{R}\right) / 2$, in the wide band limit. Finally, for the purpose of analyzing the nonlinear transport properties, one needs calculate the local spectral function of the central region, which can be defined as

$$
\begin{aligned}
A(\omega) & =-\left.i\left[G_{d}^{+-}(\omega)-G_{d}^{-+}(\omega)\right]\right|_{\lambda=0} \\
& =-\left.i \sum_{n} w_{n}\left[G_{c}^{+-}\left(\omega-n \omega_{0}\right)-G_{c}^{-+}\left(\omega+n \omega_{0}\right)\right]\right|_{\lambda=0} .
\end{aligned}
$$

\section{Expressions for FCS, Current, and Shot Noise}

Inserting all these results derived in above subsection into Eq. (9) and integrating over $\lambda_{\eta-}$ and setting $\lambda_{\eta-}=-\lambda_{\eta+}=\lambda_{\eta}$, we can yield an explicit analytical formula for the CGF of the electronic transport through a single molecular QD in presence of strong electronphonon interaction

$$
\begin{aligned}
\ln \chi(\lambda)= & \mathcal{T} \int \frac{d \omega}{2 \pi} \ln \left\{1+\sum_{n m} T_{n m}(\omega)\right. \\
& \times\left[f_{L+n}\left(1-f_{R-m}\right)\left(e^{i \lambda}-1\right)\right. \\
& \left.\left.+f_{R+m}\left(1-f_{L-n}\right)\left(e^{-i \lambda}-1\right)\right]\right\},
\end{aligned}
$$

where $T_{n m}(\omega)$ is the transmission coefficient of electron between the left and right electrodes involving vibrational quanta $n$ and $m$ :

$$
T_{n m}(\omega)=\frac{\Gamma_{L} \Gamma_{R} w_{n} w_{m}}{\mathcal{D}_{0}(\omega)}
$$

with $\lambda \equiv \lambda_{L}-\lambda_{R}, \mathcal{D}_{0}(\omega)=\left.\mathcal{D}_{\lambda}(\omega)\right|_{\lambda=0}$ and $f_{\eta \pm n}$ is a shorthand for $f_{\eta}\left(\omega \pm n \omega_{0}\right)$.

It is known that one of the advantages of the FCS conception in quantum transport is that the FCS expression can be used to distinguish the elementary events of electronic tunneling, thus provide some insight into the 
relevant transport properties $\frac{54}{4}$ Therefore, we can conclude from Eq. (33) that under the condition of weak tunneling and strong EPI, electronic transport through a molecular QD can still be regarded as three distinct independent processes: (i) electrons transmitted from the left electrode to the right with probability $P_{+}=$ $\sum_{n m} T_{n m} f_{L+n}\left(1-f_{R-m}\right)$; (ii) transmission from right to left with $P_{-}=\sum_{n m} T_{m n} f_{R+n}\left(1-f_{L-m}\right)$; (iii) no transmission with $P_{0}=1-P_{+}-P_{-}$. Accordingly, the generating function for each process is $\chi=\sum_{\xi=+,-, 0} P_{\xi} X_{\xi}$ with $X_{\xi}=e^{i \xi \lambda}$. It is worth to notice that these transmission processes involve all possible phonon-assisted events. For example, the independent process (i) describes the specific electronic tunneling that an electron with energy $\omega$ in the left lead absorbs $n$ (if $n \geq 0$ ) or emits $n$ (if $n<0$ ) phonon in the left bridge, and tunnels through the central region, and eventually enters into the right lead with emitting $m$ (if $m \geq 0$ ) or absorbing $m$ (if $m<0$ ) phonon in the right bridge. Bearing in mind of these considerations, it can be addressed that the present FCS formula Eq. (33) is a direct extension of the original LevitovLesovik formula,, 42

$$
\begin{aligned}
\ln \chi(\lambda)= & \mathcal{T} \int \frac{d \omega}{2 \pi} \ln \left\{1+T(\omega)\left[f_{L}\left(1-f_{R}\right)\left(e^{i \lambda}-1\right)\right.\right. \\
& \left.\left.+f_{R}\left(1-f_{L}\right)\left(e^{-i \lambda}-1\right)\right]\right\},
\end{aligned}
$$

to the inelastic electron transfer processes with either absorption or emission of phonon.

Noticing the relation $w_{-n}=e^{-n \omega_{0} / T} w_{n}$, we can further deduce from Eq. (33) that in the present approximation, the FCS cumulants obey a universal relation

$$
\chi(V, \lambda)=\chi(V,-\lambda+i V / T)
$$

which means that the detailed balance condition between the probabilities of opposite number of particles transferred through the QD remain valid even in the presence of electron-vibration interaction $\sqrt[54,55]{ }$ The out-ofequilibrium fluctuation relations relate current correlation functions at any order at equilibrium to response coefficients of current cumulants of lower order $\underline{\underline{54,55}}$

Based on the explicit analytical expression Eq. (33) of CGF, one can obtain all cumulants of charge transfer distribution through the molecular QD. We will however focus on the investigation of the first two cumulants, i.e., the average current through the system and the zerofrequency shot noise, in this paper, because they are the most easily accessible quantities in the experimental measurements. In specific, the average current $I$ from the left lead to the QD is evaluated as follows:

$$
\begin{aligned}
I= & \left.\frac{2 e}{\hbar} \frac{1}{\mathcal{T}} \frac{\partial \ln \chi(\lambda)}{\partial\left(i \lambda_{L}\right)}\right|_{\lambda=0}=\frac{2 e}{h} \int d \omega \sum_{n m} T_{n m}(\omega) \\
& \times\left[f_{L+n}\left(1-f_{R-m}\right)-f_{R+m}\left(1-f_{L-n}\right)\right]
\end{aligned}
$$

From Eq. (37) the current can be separated as two contributions of elastic and inelastic parts, $I=I_{e l}+I_{i n}$, where the elastic current is

$$
I_{e l}=\frac{2 e \Gamma_{L} \Gamma_{R}}{h} \int d \omega \frac{w_{0}^{2}}{\mathcal{D}_{0}(\omega)}\left[f_{L}(\omega)-f_{R}(\omega)\right]
$$

While the zero-frequency current fluctuation $S$ is give by:

$$
\begin{aligned}
S=\left.\frac{4 e^{2}}{\hbar} \frac{1}{\mathcal{T}} \frac{\partial^{2} \ln \chi(\lambda)}{\partial\left(i \lambda_{L}\right)^{2}}\right|_{\lambda=0}= & \frac{4 e^{2}}{h} \int d \omega\left(\sum_{n m} T_{n m}(\omega)\left[f_{L+n}\left(1-f_{R-m}\right)+f_{R+m}\left(1-f_{L-n}\right)\right]\right. \\
& \left.-\left\{\sum_{n m} T_{n m}(\omega)\left[f_{L+n}\left(1-f_{R-m}\right)-f_{R+m}\left(1-f_{L-n}\right)\right]\right\}^{2}\right)
\end{aligned}
$$

Before ending this section, we compare our CGF formula Eq. (33) with the previous PTA result, Eq. (2) in Ref. 51, which is obtained under the same limitation conditions, strong EPI and weak tunnel-coupling. As mentioned above, the PTA scheme takes no account of vibrational effect in the tunneling self-energy in its Dyson equation for calculating the full electronic GF $G_{d}$. One can argue that the PTA only considers virtual excitation of phonon in each electronic tunneling process, i.e., when an electron tunnels onto the molecule it excites the local phonon and fully de-excites the phonon upon leaving the dot. Therefore the electron after tunneling has the same energy as that before tunneling. This is why the PTA CGF [Eq. (22) in Ref. 51] has the similar form with the original Levitov-Lesovik formula Eq. (35). While in the present approximation, after an electron tunnels into the molecular QD and excites the phonon, a virtual tunneling of electron into the leads is considered leading to excitations of particle-hole pairs in the leads. Then the electron tunnels out of the molecular QD and de-excites the phonon, but some particle-hole pairs remain in the leads, therefore energies of the electron before and after tunneling can be different. Physically, our results seem more reasonable because elastic and inelastic tunneling 
processes are both considered while only elastic tunneling processes are considered in the PTA scheme.

\section{RESULTS AND DISCUSSIONS}

Here we carry out the numerical calculation of the current and zero-frequency shot noise through a singlemolecular QD using Eqs. (37) and (39). For simplicity, we consider the system with symmetric tunnel-couplings to the leads, $\Gamma_{L}=\Gamma_{R}=0.1 \omega_{0}, \Gamma=\Gamma_{L}+\Gamma_{R}$, and assume the bias voltage is applied symmetrically, i.e., $\mu_{L / R}=\mu \pm V / 2$. Therefore we can only consider positive bias voltage $V \geq 0$ in the following calculations. We also set the phonon energy $\omega_{0}=1$ as the unit of energy throughout the rest of the paper and choose the Fermi levels of the two leads as the reference of energy $\mu_{L}=\mu_{R}=\mu=0$ at equilibrium. The normalized EPI constant is set to be $g=1$ to ensure the validate of the approximation scheme involved in the present paper.

Below we mainly consider zero temperature, at which the weighting factor becomes

$$
w_{n}= \begin{cases}e^{-g^{2}} g^{2 n} / n !, & n \geq 0 \\ 0, & n<0\end{cases}
$$

meaning that only phonon emission processes are allowed.

\section{A. Self energy and spectral function}

We first examine the dependence of the tunnelinginduced electronic self-energy, Eq. (31), on the bias voltage in Fig. 2 at zero temperature. We find that its imaginary part has explicit stepwise structures in frequency domain related to the opening of the inelastic channels, and the widths and heights of these steps are controlled by external applied bias voltage. Correspondingly, the real part of the self-energy shows multi-peaks with logarithmic singularities due to the Kramers-Kronig relations, which can be traced back to a previous work on EPI system by Engelsberg and Schrieffer for bulk Einstein phonons in 1963.56 It is observed that the real parts of the self-energies, i.e., the values of the energy shift, are relatively small in the case of weak tunnel-coupling.

We then calculate the equilibrium spectral function Eq. (32) for the systems with $\widetilde{\varepsilon}_{d}=0$ and $0.5 \omega_{0}$. As shown in Fig. 3, one can find that the main effects of the electron-phonon coupling is the appearance of the phonon-assisted side peaks in the spectral function. At the zero temperature case and the renormalized level $\widetilde{\varepsilon}_{d}=0$, the main resonant peak at $\omega=0$ is Lorentzian in shape, while the phonon side peaks exhibit nonLorentzian form due to stepwise jumps in the imaginary part of the self-energy as depicted in Fig. 2(a). Peculiarly, these phonon side peaks symmetrically distribute in both sides of the energy axes at $\omega= \pm|n| \omega_{0}$ with
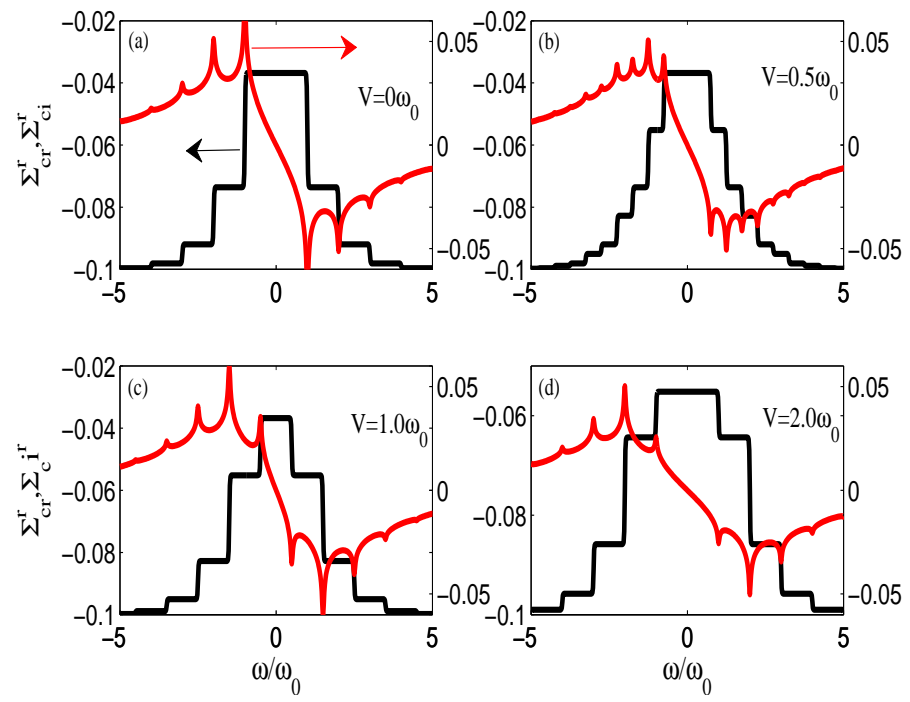

Figure 2: (Colour online) The real part (red line) and imaginary part (black line) of the vibration-modified retarded selfenergies are plotted for different bias-voltages, $V=0$ (a), $0.5 \omega_{0}(\mathrm{~b}), 1.0 \omega_{0}(\mathrm{c})$, and $2.0 \omega_{0}(\mathrm{~d})$, respectively, at zero temperature. The parameters used for calculation are taken as: $\Gamma_{L}=\Gamma_{R}=0.1 \omega_{0}, g=1.0$.
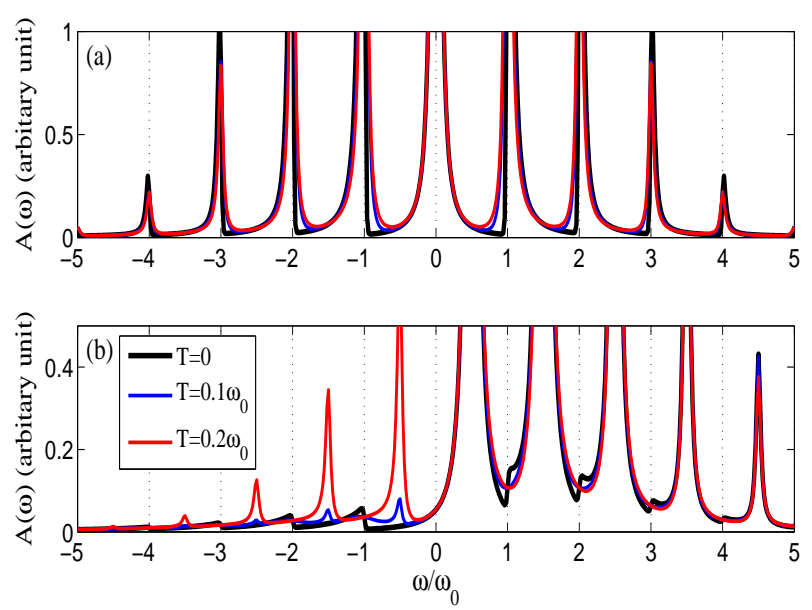

Figure 3: (Colour online) The equilibrium calculated spectral function of the QD as a function of the energy $\omega$ for (a) $\widetilde{\varepsilon}_{d}=0$ and (b) $\widetilde{\varepsilon}_{d}=0.5 \omega_{0}$ at different temperatures $T=0,0.1 \omega_{0}$, and $0.2 \omega_{0}$, respectively. The remaining parameters are the same as those in Fig. 2

gradually reduced heights. This behavior can be understood from the local spectral function Eq. (32) 33 At zero temperature, the local spectral function has two contributions, the lesser GF $G_{c}^{-+}\left(\omega+n \omega_{0}\right)$ and the greater GF $G_{c}^{+-}\left(\omega-n \omega_{0}\right)$ at $n \geq 0$. These two GFs correspond to the local electron and hole propagators, respectively, and thus are proportional to the occupation number $n_{d}$ for the QD electron or $1-n_{d}$ for the hole. For the system with $\widetilde{\varepsilon}_{d}=0$ and symmetrical tunnel-couplings to electrodes $\Gamma_{L}=\Gamma_{R}$, the QD is partially occupied by electrons, $n_{d}=1 / 2$. One can therefore interpret that 
the phonon side peaks at negative $\omega$ region result from the phonon emission by local electrons while the phonon side peaks at positive $\omega$ region originate from the phonon emission by local holes.

When $\widetilde{\varepsilon}_{d}$ is far away from the the chemical potentials $\mu_{L}=\mu_{R}=\mu=0$, the side peaks become asymmetry on the two sides of the main peak located at $\omega=\widetilde{\varepsilon}_{d}$. For example, the spectral function of the system with $\widetilde{\varepsilon}_{d}=0.5 \omega_{0}$ exhibits Lorentzian-type phonon side peaks only at positive $\omega$ region, $\omega=\widetilde{\varepsilon}_{d}+|n| \omega_{0}$, but no phonon side peak at negative $\omega$ region, because no electron occupies the $\mathrm{QD}, n_{d} \simeq 0$. More interestingly, a small abrupt jump in the spectral function survives at $\omega= \pm|n| \omega_{0}$ as depicted in Fig. 3(b), which is also stemming from the stepwise jumps in the imaginary part of the self-energy occurring at these frequencies corresponding to the opening of inelastic scattering processes. It is not surprise that with raising temperature $T$, all these novel features in the spectral function are gradually smoothed away. Besides, several phonon side peaks reemerge in negative energy regions due to the opening of phonon absorption channels at higher temperature [Fig. 3(b)]. Furthermore, application of external bias voltage will change the occupation number of electrons at the QD, and will inevitably change the spectral function. It will be shown below that it is the complex dependences of the self-energy on the bias voltage in conjunction with the tiny features in the spectral function $A(\omega)$ that determines exotic properties of the nonlinear conductance and shot noise.

\section{B. Current and differential conductance}

Before investigating nonlinear transport, we consider the zero-temperature linear conductance at first. It is easy from Eq. (37) to yield

$$
G=\left.\frac{d I}{d V}\right|_{V=0}=\frac{\Gamma_{L} \Gamma_{R} w_{0}^{2}}{\left[\widetilde{\varepsilon}_{d}+\Sigma_{c r}^{r}(0)\right]^{2}+\left|\Sigma_{c i}^{r}(0)\right|^{2}},
$$

with $\Sigma_{c r}^{r}(0)=0$ and $\Sigma_{c i}^{r}(0)=-i\left(\Gamma_{L}+\Gamma_{R}\right) w_{0} / 2$. Therefore, in the linear transport regime, the effect of the strong electron-phonon interaction is just to narrow the resonance peak of the conductance due to the FranckCondon blockade. Besides, the linear conductance exhibits no phonon sidebands as a function of the gate voltage. These two aspects of the linear conductance are in good agreement with the previous results for weak electron-phonon coupling systems based on the perturbative calculation up to the second-order of the electronphonon coupling constant, $g^{2} .24$

The situation is very different for the nonlinear transport as shown in Fig. 4, in which we plot the currents $I$ and corresponding differential conductances $d I / d V$ as functions of bias voltage $V>0$ for the systems with $\widetilde{\varepsilon}_{d}=0$ and $0.5 \omega_{0}$ at zero temperature. For the purpose of analysis, we also plot their corresponding elastic and inelastic parts. It is easy to obtain from Eq. (37) that only
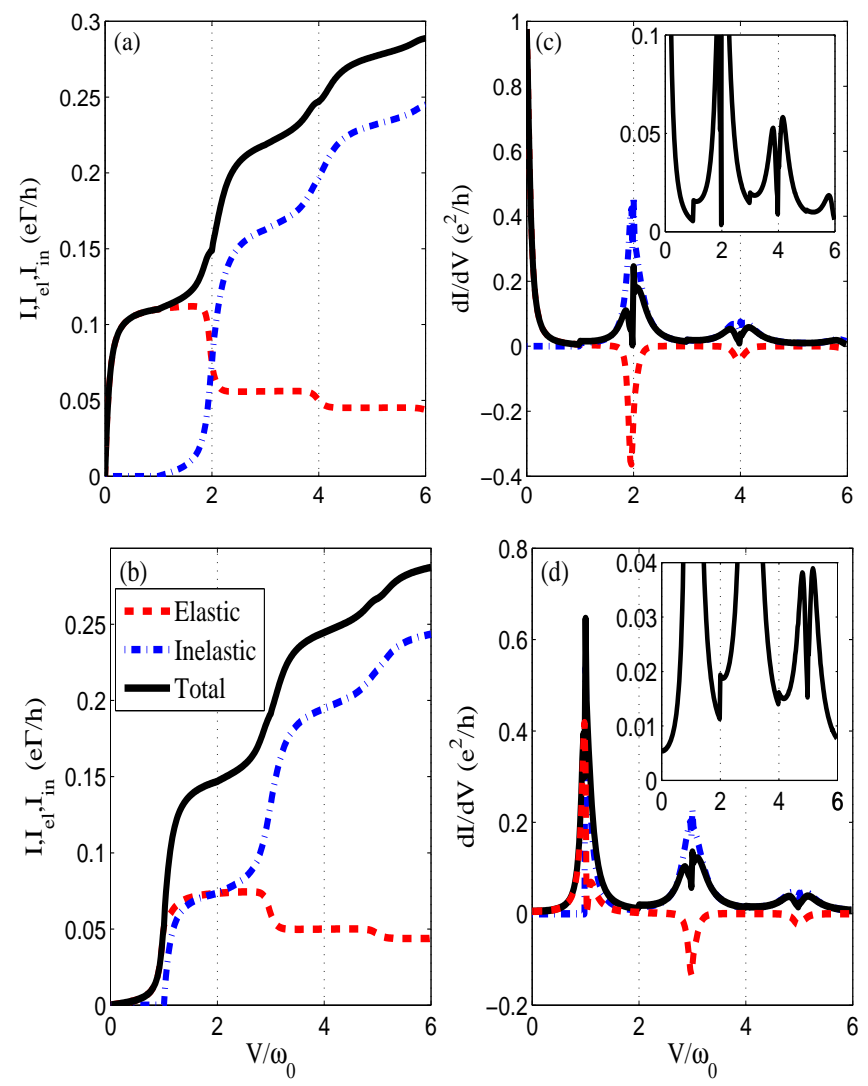

Figure 4: (Colour online) (a, b) The calculated total current (solid line), elastic current (dashed line), inelastic current (dotted-dashed line); and (c, d) the corresponding differential conductances as functions of bias voltage for a singlemolecular QD with $\widetilde{\varepsilon}_{d}=0(\mathrm{a}, \mathrm{c})$ and $0.5 \omega_{0}(\mathrm{~b}, \mathrm{~d})$, respectively, at zero temperature. The remaining parameters are the same as those in Fig. 2 The inset shows the enlarged tiny features of the respective differential conductances. (See text for details).

when the bias voltage is larger than the phonon energy, $V \geq \omega_{0}$, the inelastic current channels are opening, which leads to abrupt upward jumps of the differential conductance at $V=n \omega_{0}(n>0)$. Nevertheless, these upward jumps can be divided into two sorts, big jumps and tiny jumps. To obtain clear interpretation of these jumps, we give an explicit expression of the main contributive terms of the inelastic current at zero temperature as:

$$
I_{i n} \simeq \frac{2 e}{h} \Gamma_{L} \Gamma_{R} w_{0} w_{1} \int_{\omega_{0}-V / 2}^{V / 2} d \omega\left[\frac{1}{\mathcal{D}_{0}(\omega)}+\frac{1}{\mathcal{D}_{0}(-\omega)}\right]
$$

For the partially filled QD $\left(\widetilde{\varepsilon}_{d}=0\right)$, the external bias voltage $V=\omega_{0}$ causes only a tiny jump due to the nonzero value of $\mathcal{D}_{0}( \pm V / 2) \simeq\left(\omega_{0} / 2\right)^{2}+\left|\Sigma_{c i}^{r}\left( \pm \omega_{0} / 2\right)\right|^{2}$, but the bias voltage $V=2 \omega_{0}$ results in a big jump owing to the minimum value in $\mathcal{D}_{0}\left( \pm \omega_{0} \mp V / 2\right) \simeq\left|\Sigma_{c i}^{r}(0)\right|^{2}$; while for the empty QD $\left(\widetilde{\varepsilon}_{d}=0.5 \omega_{0}\right)$, big jumps will occur at $V=\omega_{0}$ and $3 \omega_{0}$ because of $\mathcal{D}_{0}(\omega) \simeq\left(\omega-\omega_{0} / 2\right)^{2}+$ $\left|\Sigma_{c i}^{r}(\omega)\right|^{2}$. The tiny jumps at $V=2 \omega_{0}$ and $4 \omega_{0}$ are the 
remaining effect of the small abrupt jump in the spectral function as shown in Fig. 3(b).

Now we turn to discuss the elastic part of the tunneling current. The elastic current formula Eq. (38) can be simplified at zero temperature as

$$
I_{e l}=\frac{2 e}{h} \Gamma_{L} \Gamma_{R} w_{0}^{2} \int_{-V / 2}^{V / 2} d \omega \frac{1}{\mathcal{D}_{0}(\omega)} .
$$

As usually, the elastic current rises monotonously as the bias voltage of the left lead is increasing up to the energy level of the QD, $V=2 \widetilde{\varepsilon}_{d}=0$ or $1.0 \omega_{0}$, i.e., the resonant tunneling condition is reached. It is quite surprise, however, that the elastic current exhibits decrease steps with increasing further the bias voltages. To give an underlying interpretation of this decrease, we examine the derivative of the elastic current with respect to the bias voltage. Differentiating Eq. (43) with respect to $V$, the nonlinear conductance can be written as $g^{e l}=d I_{e l} / d V=g_{1}^{e l}+g_{2}^{e l}$, with

$$
g_{1}^{e l}=\frac{e^{2}}{h} \Gamma_{L} \Gamma_{R} w_{0}^{2}\left[\frac{1}{\mathcal{D}_{0}(-V / 2)}+\frac{1}{\mathcal{D}_{0}(V / 2)}\right],
$$

and

$$
\begin{aligned}
g_{2}^{e l} \simeq & -\frac{2 e^{2}}{h} \Gamma_{L} \Gamma_{R} w_{0}^{2} \sum_{n=1}^{\infty} w_{n} \int_{-V / 2}^{V / 2} d \omega \frac{\left|\Sigma_{c i}^{r}(\omega)\right|}{\mathcal{D}_{0}^{2}(\omega)} \\
& \times\left[\Gamma_{L} \delta\left(\omega-n \omega_{0}+V / 2\right)+\Gamma_{R} \delta\left(\omega+n \omega_{0}-V / 2\right)\right] .
\end{aligned}
$$

The first term, $g_{1}^{e l}$, is proportional to the transmission probability $T_{00}(V / 2)$ and results in the first resonant peak at $V=2 \widetilde{\varepsilon}_{d}$; while the second term, $g_{2}^{e l}$, is stemming from the bias-voltage-dependent self-energy and it always makes negative contribution and becomes predominant over the first term at $V=2 n \omega_{0}+2 \widetilde{\varepsilon}_{d}(n>0)$ and at $V=2 n \omega_{0}-2 \widetilde{\varepsilon}_{d}(n>1)$, which is responsible for decrease steps in the elastic current and the double-peak structure in the total differential conductance at $V=2 \omega_{0}, 4 \omega_{0}$ for the QD with $\widetilde{\varepsilon}_{d}=0$ or at $V=3 \omega_{0}, 5 \omega_{0}$ for $\widetilde{\varepsilon}_{d}=0.5 \omega_{0}$.

It should be noted that the inelastic scattering induced discontinuities, i.e., downward or upward steps, in the differential conductance have been previously reported based on the self-consistent Born approximation and the second-order perturbation calculations in the case of weak EPI $20,23,24$ Our present nonperturbative calculations show more complex behavior for the systems with strong EPI and hard phonon $\omega_{0} \gg \Gamma$ : tiny upward steps and double-peak profiles.

All these tiny features in the differential conductance will be inevitably smeared away with increasing temperature, but those big jumps will survive (not shown here). Therefore, the differential conductance will still reflect the main profile of the spectral function of the molecular QD as shown in Fig. 3 at relatively high temperature. Besides, it is observed that the magnitudes of these big jumps gradually decrease with increasing bias voltage due to Franck-Condon blockade.

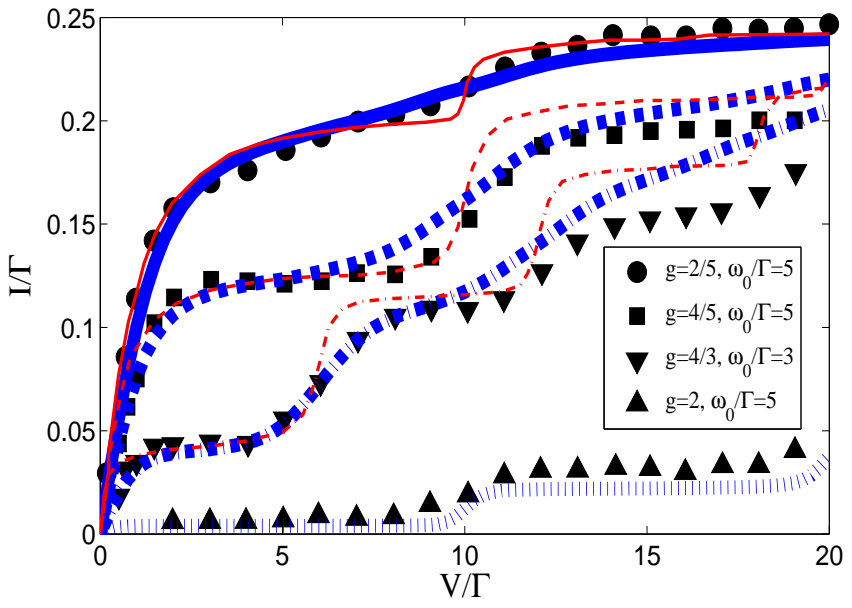

Figure 5: (Colour online) The calculated current as functions of bias voltage for $\widetilde{\varepsilon}_{d}=0$ at the temperature $T=0.2 \Gamma$. The thick-blue lines denote the present calculations, the thin-red lines are those of the PTA, and the discrete symbols represent the diagrammatic Monte Carlo data. Circles stand for the QD with $g=2 / 5, \omega_{0} / \Gamma=5$; squares for $g=4 / 5, \omega_{0} / \Gamma=$ 5 ; downward triangles for $g=4 / 3, \omega_{0} / \Gamma=3$; and upward triangles for $g=2, \omega_{0} / \Gamma=5$.

Before turning to discuss the shot noise, in order to estimate the quality of the present approximation, we compare our results with those of accurate diagrammatic Monte Carlo simulation,, 38 by plotting the calculated $I-V$ characteristics for several different molecular QD systems at a finite temperature $T=0.2 \Gamma$, as shown in Fig. 5 . For comparison, we also plot the results calculated using PTA. It is clear that in the regime of moderate to large bias voltage $V$, our method exhibits better consistency with the Monte Carlo simulation than the PTA.

\section{Zero-frequency shot noise}

In what follows, we analyze the zero-frequency shot noise at zero temperature, which can be calculated using a simplified expression according to the Eq. (39)

$$
\begin{aligned}
S= & 2 e I-\frac{4 e^{2}}{h}\left(\Gamma_{L} \Gamma_{R}\right)^{2} \sum_{n m n^{\prime} m^{\prime}} w_{n} w_{m} w_{n^{\prime}} w_{m^{\prime}} \\
& \times \int_{\omega_{1}}^{\omega_{2}} d \omega \frac{1}{\mathcal{D}_{0}^{2}(\omega)},
\end{aligned}
$$

with $\omega_{1}=\max \left(n \omega_{0}-V / 2, n^{\prime} \omega_{0}-V / 2\right)$ and $\omega_{2}=$ $\min \left(V / 2-m \omega_{0}, V / 2-m^{\prime} \omega_{0}\right)$. We can also separate the shot noise as two contributions of elastic and inelastic parts, $S=S_{e l}+S_{i n}$, with the elastic part being

$$
S_{e l}=2 e I_{e l}-\frac{4 e^{2}}{h}\left(\Gamma_{L} \Gamma_{R}\right)^{2} w_{0}^{4} \int_{-V / 2}^{V / 2} d \omega \frac{1}{\mathcal{D}_{0}^{2}(\omega)} .
$$

In Figs. 6(a) and (c), we plot the calculated shot noise and its two contributive parts as functions of bias voltage $V>0$ for the systems with $\widetilde{\varepsilon}_{d}=0$ and $\widetilde{\varepsilon}_{d}=0.5 \omega_{0}$, 
respectively. It is observed that for the empty QD $\left(\widetilde{\varepsilon}_{d}=0.5 \omega_{0}\right)$, the shot noise of the elastic channel inherits the same behavior as the elastic current with increasing bias voltage, continuous increase up to the resonant point and downward steps at $V=3 \omega_{0}$ and $5 \omega_{0}$. On the contrary, the inelastic shot noise exhibits abrupt downward jumps at $V=\omega_{0}$ and $3 \omega_{0}$, instead of upward jumps in the inelastic current. At $V=\omega_{0}$, we can evaluate approximately the correction to the shot noise due to the inelastic tunneling for the system with $\widetilde{\varepsilon}_{d}=0.5 \omega_{0}$ as

$$
\begin{aligned}
S_{i n} \simeq & 2 e I_{i n}-\frac{4 e^{2}}{h}\left(\Gamma_{L} \Gamma_{R}\right)^{2} w_{0}^{2} w_{1}\left(2 w_{0}+w_{1}\right) \\
& \times \int_{\omega_{0}-V / 2}^{V / 2} d \omega\left[\frac{1}{\mathcal{D}_{0}^{2}(\omega)}+\frac{1}{\mathcal{D}_{0}^{2}(-\omega)}\right] \\
\simeq & \frac{4 e^{2}}{h} \frac{\Gamma_{L} \Gamma_{R} w_{0} w_{1}}{\left|\Sigma_{c i}^{r}(0)\right|^{2}} \frac{\left(\Gamma_{L}-\Gamma_{R}\right)^{2}-4 \Gamma_{L} \Gamma_{R}\left(1+\frac{w_{1}}{w_{0}}\right)}{\left(\Gamma_{L}+\Gamma_{R}\right)^{2}}
\end{aligned}
$$

For the symmetric tunnel-coupling case considered in this paper, $\Gamma_{L}=\Gamma_{R}$, the opening of inelastic channel generates a negative contribution to the shot noise. The same corrections of the inelastic noise will be predicted at $V=3 \omega_{0}$ and $5 \omega_{0}$, leading to downward jumps in the shot noise in association with the elastic noise. While the situation is more complex for the partially filled QD $\left(\widetilde{\varepsilon}_{d}=0\right)$. At first, the inelastic noise shows a tiny upward jump at $V=\omega_{0}$, i.e. a positive correction, because of

$$
\begin{aligned}
S_{i n} \simeq & \frac{4 e^{2}}{h} \frac{\Gamma_{L} \Gamma_{R} w_{0} w_{1}}{\left[\left(\omega_{0} / 2\right)^{2}+\left|\Sigma_{c i}^{r}\left(\omega_{0} / 2\right)\right|^{2}\right]^{2}} \\
& \times\left[\left(\omega_{0} / 2\right)^{2}+\left|\Sigma_{c i}^{r}\left(\omega_{0} / 2\right)\right|^{2}-\Gamma_{L} \Gamma_{R} w_{0}^{2}\left(2+\frac{w_{1}}{w_{0}}\right)\right] .
\end{aligned}
$$

But the inelastic noise becomes downward jump at $V=$ $2 \omega_{0}$ again. Actually, the inelastic noise contribution has been examined for a QD with weak EPI, a soft phonon $\omega_{0} \ll \Gamma$, and arbitrary transmission based on the secondorder perturbative calculation at $V=\omega_{0}$ where the inelastic channel is just opening $\stackrel{45}{ }$ A sign change in the inelastic noise correction at certain domains in parameter space of transmission and energy level has been addressed and ascribed to the underlying competition between elastic and inelastic processes. Very recently, the negative contribution to noise has been experimentally observed on $\mathrm{Au}$ nanowires in the weak EPI limit and has been ascribed to the coherent two-electron tunneling processes assisted by phonon emission that reduce electronic fluctuations due to Pauli principle. 46 The present investigation in this paper indicates indeed that the interplay of elastic and inelastic scattering processes causes the following properties of shot noise: (1) the elastic shot noise exhibits a downward step at the bias voltages $V=2\left(n \omega_{0} \pm \widetilde{\varepsilon}_{d}\right)>0$ as the elastic current does; (2) Meanwhile, the opening of inelastic channel at these bias voltages induces an abrupt increase of the transmission probability of the inelastic channel [i.e. the inelastic current as shown in the above subsection, Figs. 4(a) and (b)] and consequentially results in an obvious downward jump; otherwise the inelastic noise shows only a tiny increase; (3) In particular, for the molecular QD with an energy level of $\widetilde{\varepsilon}_{d}=0.5 \omega_{0}$, the inelastic noise becomes negative at $V=2\left(\omega_{0}-\widetilde{\varepsilon}_{d}\right)=\omega_{0}$, i.e. the sign change of the inelastic correction to shot noise in the case of strong EPI and a hard phonon. Nevertheless, no such negative correction to noise was found in the PTA calculations. 51 We argue that this is because the PTA considers only the elastic scattering processes as pointed out above.
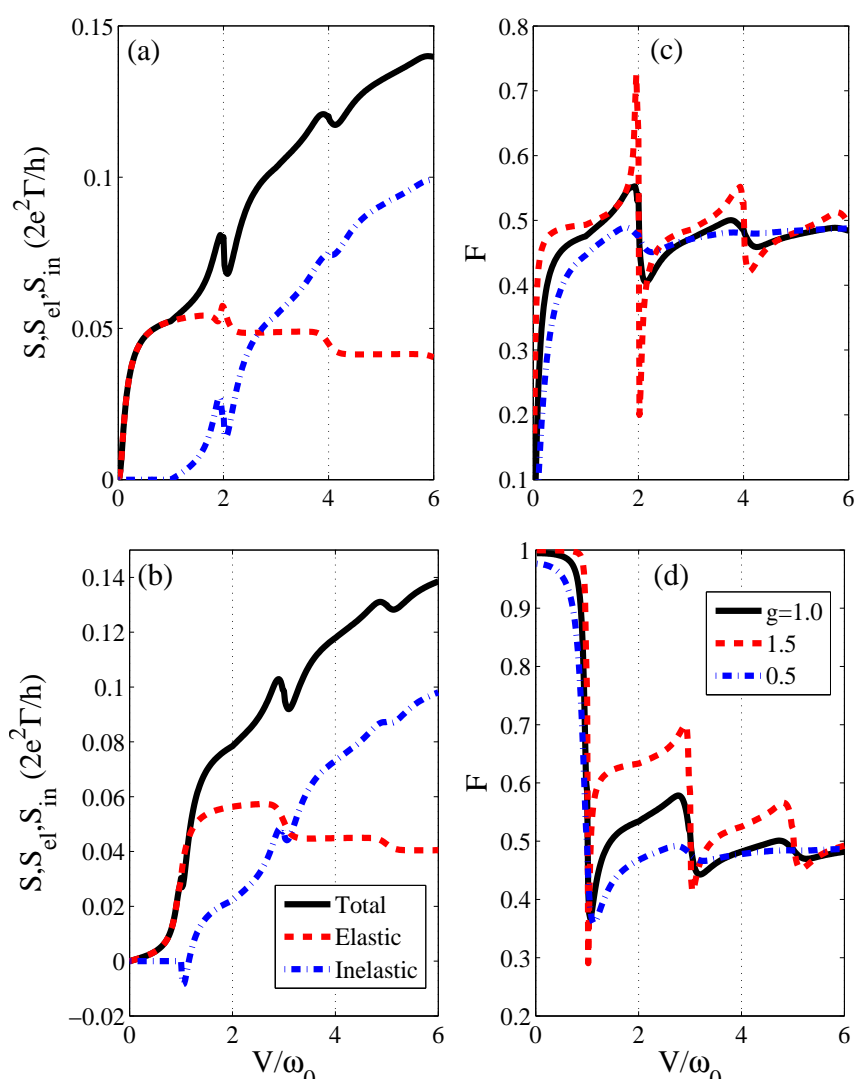

Figure 6: (Colour online) (a, b) The zero-temperature shot noise (solid line), and its elastic (dashed line) and inelastic (dotted-dashed line) parts as functions of bias voltage for a single-molecular QD with $\widetilde{\varepsilon}_{d}=0$ (a) and $0.5 \omega_{0}$ (b), respectively. The electron-phonon coupling constant is set to be $g=1.0 ;(\mathrm{c}, \mathrm{d})$ The corresponding Fano factors for the two systems, $\widetilde{\varepsilon}_{d}=0$ (c) and $0.5 \omega_{0}(\mathrm{~d})$, with different electronphonon coupling constants $g=1.0$ (solid line), 1.5 (dashed line), and 0.5 (dotted-dashed line). We set $\Gamma=0.1 \omega_{0}$ in the calculations.

To analyze the relative strength of noise, a more useful quantity is the so-called Fano factor $F$ defined as the ration of the shot noise to the Poisson value, $F=$ $S / 2 e I$. It is obvious from Eq. (46) that the present approximation exhibits no super-Poissonian noise, being in agreement with the previous NGF calculation in Ref. 51 under the same approximation, strong electronphonon interaction and thermal equilibrated phonon. It also deserves to point out that the present result is in no conflict with that of the rate-equation calculations. 
Even though a giant Fano factor has been predicted due to avalanchelike transport of electrons by rate-equation calculations, $\frac{11,12}{1}$ it has been subsequently clarified that a single-level molecular QD will exhibit super-Poissonian noise only when both of two conditions, external-biasvoltage-driven unequilibriated phonon and asymmetric tunnel-couplings between the QD and two leads, are simultaneously satisfied $\stackrel{14,15}{=}$ Otherwise, the shot noise will decrease with increasing strength of dissipation of the hot phonon to environment, and eventually become subPoissonian noise and show steplike behavior. ${ }^{15,16}$ In this paper, our NGF calculations predict more rich oscillatory behavior of the Fano factor as a function of the bias voltage, as shown in Figs. 6(c) and (d). It is interesting to observe that the aforementioned downward jumps in the shot noise in conjunction with the upward steps in the current induce obvious dips in the Fano factor, whose values can be smaller than $1 / 2$. Since for a resonant tunneling model with a small tunneling rate $\Gamma$, the typical value of the Fano factor of a symmetric tunneljunction at large bias voltage is right equal to $1 / 2$, this unusual smaller-than-one-half Fano factor therefore can be regarded as an unambiguous signature of vibronic participation in electronic tunneling.

\section{CONCLUSION}

In conclusion, in this paper we have investigated inelastic effects on the FCS of electronic tunneling through a single-molecular QD in the presence of strong electronphonon interaction, weak tunnel-couplings, and hard phonon mode. For this purpose, we have performed the Lang-Firsov canonical transformation for the local electron-phonon interaction and made use of the noncrossing approximation to decouple the electronic and phononic degrees of freedom. Then we have employed the generalized nonequilbirium Green function technique for the FCS and derived an explicit analytical LevitovLesovik formula for the cumulant generating function under the approximation that the the molecular vibration is assumed to be always thermally equilibrated due to fast dissipation to a thermal phonon bath, i.e. the environment. This formula can not only provide fundamental knowledge of how to clarify independent elementary processes in the vibration-assisted charge transfer, but also give analytical expressions for the tunneling current and its zero-frequency shot noise. Subsequently, we have carried out numerical calculations for the current and shot noise of a QD with symmetric tunnel-couplings at zero temperature and further analyzed their bias-voltage dependence in detail.

Even though several of our formal results, for example, the upward or downward jumps in the current and shot noise only at $V=\omega_{0}$, were already addressed in previous papers by the second-order perturbative calculations for weak EPI system, $11,23,24,45$ there are still some debates in these issues in the literature. The present paper has provided complementary investigation for strong EPI system. We have found that: (i) The singularities in the electronic self-energy and spectral function cause discontinuities in the zero-frequency shot noise in the weak tunnel-coupling case, i.e. weak bare elastic transparency of the molecular junction. The sign of the discontinuity occurring at $V=\omega_{0}$ (single-phonon scattering process) depends on the normalized energy level of the molecular QD. For an empty QD, $\widetilde{\varepsilon}_{d}=0.5 \omega_{0}$, the inelastic channel provides a negative contribution to noise at $V=\omega_{0}$; otherwise, a positive contribution is observed. Moreover, multi-phonon scattering events will always induce downward jumps. It is noticed that the opening of inelastic channel can also affect the elastic channel, leading to downward steps in the elastic part of the current and shot noise; (ii) Contrary to the results of rate-equation calculations, our investigations predict oscillatory structure and apparent dips in the Fano factor. The small Fano factor, $F<1 / 2$, can be considered as a typical characteristics of phonon-assisted electronic tunneling through a single molecular junction.

Noticeably, our approximative calculations for the strong EPI system with an equilibrated phonon have reproduced the logarithmic singularities in the electronic self-energies 56 and consequently found the discontinuities in the differential conductance and shot noise. It is therefore desirable in the future research to develop a fully self-consistent calculation, i.e. solving the coupled Dyson equations for the electronic $\mathrm{GF} G_{c}\left(t, t^{\prime}\right)$ and the phononic GF $K\left(t, t^{\prime}\right)$ simultaneously, to observe the unequilibrated phonon effect on the singularities and discontinuities.

\section{Acknowledgments}

This work was supported by Projects of the National Basic Research Program of China (973 Program) under Grant No. 2011CB925603, and the National Science Foundation of China, Specialized Research Fund for the Doctoral Program of Higher Education (SRFDP) of China.
1 H. Park, J. Park, A. Lim, E. Anderson, A. Allvisatos, and P. McEuen, Nature 407, 57 (2000).

2 J. Park, A.N. Pasupathy, J.I. Goldsmith, C. Chang, Y. Yaish, J.R. Petta, M. Rinkoski, J.P. Sethna, H. Abruna,
P.L. McEuen, and D.C. Ralph, Nature 417, 722 (2002).

3 N.B. Zhitenev, H. Meng, and Z. Bao, Phys. Rev. Lett. 88, 226801 (2002).

4 E.M. Weig, R.H. Blick, T. Brandes, J. Kirschbaum, W. 
Wegscheider, M. Bichler, and J.P. Kotthaus, Phys. Rev. Lett. 92, 046804 (2004).

${ }^{5}$ L.H. Yu, Z.K. Keane, J.W. Ciszek, L. Cheng, M.P. Stewart, J.M. Tour, and D. Natelson, Phys. Rev. Lett. 93, 266802 (2004); L.H. Yu and D. Natelson, Nano Lett. 4, 79 (2004).

6 A.N. Pasupathy, J. Park, C. Chang, A.V. Soldatov, S. Lebedkin, R.C. Bialczak, J.E. Grose, L.A.K. Donev, J.P. Sethna, D.C. Ralph, and P.L. McEuen, Nano Lett. 5, 203 (2005).

7 B.J. LeRoy, S.G. Lemay, J. Kong, and C. Dekker, Nature 432, 371 (2004); B.J. LeRoy, J. Kong, V.K. Pahilwani, C. Dekker, and S.G. Lemay, Phys. Rev. B 72, 075413 (2005).

8 S. Sapmaz, P. Jarillo-Herrero, Ya.M. Blanter, C. Dekker, and H.S.J. van der Zant, Phys. Rev. Lett. 96, 026801 (2006); S. Sapmaz, P. Jarillo-Herrero, Ya.M. Blanter, and H.S.J. van der Zant, New J. Phys. 7, 243 (2005).

9 D. Bose and H. Schoeller, Europhys. Lett. 54, 668 (2001).

10 K.D. McCarthy, N. Prokof'ev, and M.T. Tuominen, Phys. Rev. B 67, 245415 (2003).

11 A. Mitra, I. Aleiner, and A.J. Millis, Phys. Rev. B 69, 245302 (2004); Phys. Rev. Lett 94, 076404 (2005).

12 J. Koch and F. von Oppen, Phys. Rev. Lett. 94, 206804 (2005); J. Koch and F. von Oppen, Phys. Rev. B 72, 113308 (2005).

13 J. Koch, M.E. Raikh, and F. von Oppen, Phys. Rev. Lett. 95, 056801 (2005).

14 A. Zazunov, D. Feinberg, and T. Martin, Phys. Rev. B 73, 115405 (2006).

15 X.Y. Shen, B. Dong, X.L. Lei, and N.J.M. Horing, Phys. Rev. B 76, 115308 (2007).

16 B. Dong, X.L. Lei, and N.J.M. Horing, IEEE Sensor Journal 8, 885 (2008).

17 B. Dong, H.Y. Fan, X.L. Lei, and N.J.M. Horing, J. Appl. Phys. 105, 113702 (2009).

18 R. Avriller, J. Phys.: Condens. Matter 23, 105301 (2011).

19 T. Frederiksen, M. Brandbyge, N. Lorente, and A.P. Jauho, Phys. Rev. Lett. 93, 256601 (2004); M. Paulsson, T. Frederiksen, and M. Brandbyge, Phys. Rev. B 72, 201101 (2005); T. Frederiksen, M. Paulsson, M. Brandbyge, and A.P. Jauho, Phys. Rev. B 75, 205413 (2007).

20 M. Galperin, M.A. Ratner, and A. Nitzan, J. Chem. Phys. 121, 11965 (2004).

21 J.K. Viljas, J.C. Cuevas, F. Pauly, and M. Häfner, Phys. Rev. B 72, 245415 (2005).

${ }^{22}$ L. de la Vega, A. Martín-Rodero, N. Agraït, and A. Levy Yeyati, Phys. Rev. B 73, 075428 (2006).

${ }^{23}$ R. Egger and A.O. Gogolin, Phys. Rev. B 77, 113405 (2008).

24 O. Entin-Wohlman, Y. Imry, and A. Aharony, Phys. Rev. B 80, 035417 (2009).

25 L.K. Dash, H. Ness, and R.W. Godby, J. Chem. Phys. 132, 104113 (2010); L.K. Dash, H. Ness, and R.W. Godby, Phys. Rev. B 84, 085433 (2011).

${ }^{26}$ K. Haule and J. Bonča, Phys. Rev. B 59, 13087 (1999).

27 H. Ness and A.J. Fisher, Phys. Rev. Lett. 83, 452 (1999).

28 E.G. Emberly and G. Kirczenow, Phys. Rev. B 61, 5740 (2000).

29 B. Dong, H.L. Cui, and X.L. Lei, Phys. Rev. B 69, 205315 (2004).

30 B. Dong, H.L. Cui, X.L. Lei, and N.J.M. Horing, Phys.
Rev. B 71, 045331 (2005).

31 U. Lundin and R.H. McKenzie, Phys. Rev. B 66, 075303 (2002).

32 K. Flensberg, Phys. Rev. B 68, 205323 (2003).

33 Z.Z. Chen, R. Lü, and B.F. Zhu, Phys. Rev. B 71, 165324 (2005).

34 M. Galperin, A. Nitzan, and M.A. Ratner, Phys. Rev. B 73, 045314 (2006).

35 M. Galperin, A. Nitzan, and M.A. Ratner, Phys. Rev. B 74, 075326 (2006).

36 R. Härtle, C. Benesch, and M. Thoss, Phys. Rev. B 77, 205314 (2008); R. Härtle, M. Butzin, O. Rubio-Pons, and M. Thoss, Phys. Rev. Lett. 107, 046802 (2011).

37 A. Zazunov and T. Martin, Phys. Rev. B 76, 033417 (2007).

38 L. Mühlbacher and E. Rabani, Phys. Rev. Lett. 100, 176403 (2008).

39 J. Bonča and S.A. Trugman, Phys. Rev. Lett. 75, 2566 (1995)

40 Yu.V. Nazarov, ed., Quantum Noise in Mesoscopic systems, NATO Science Series II, Vol. 97 (Kluwer, Dordrecht Boston London, 2003).

41 Ya.M. Blanter and M. Büttiker, Phys. Rep. 336, 1 (2000).

42 L.S. Levitov and G.B. Lesovik, JETP Lett. 58, 230 (1993); D.A. Ivanov and L.S. Levitov, JETP Lett. 58, 461 (1993); L.S. Levitov, H.W. Lee, and G.B. Lesovik, J. Math. Phys. 37, 4845 (1996).

43 A. Komnik and A.O. Gogolin, Phys. Rev. Lett. 94, 216601 (2005); A.O. Gogolin and A. Komnik, Phys. Rev. B 73, 195301 (2006).

44 L.S. Levitov and M. Reznikov, Phys. Rev. B 70, 115305 (2004)

45 T.L. Schmidt, and A. Komnik, Phys. Rev. B 80, 041307 (2009); R. Avriller and A. Levy Yeyati, Phys. Rev. B 80, 041309 (2009); F. Haupt, T. Novotný, and W. Belzig, Phys. Rev. Lett. 103, 136601 (2009); F. Haupt, T. Novotný, and W. Belzig, Phys. Rev. B 82, 165441 (2010).

46 M. Kumar, R. Avriller, A. Levy Yeyati, and Jan M. van Ruitenbeek, Phys. Rev. Lett. 108, 146602 (2012).

47 R. Avriller and T. Frederiksen, Phys. Rev. B 86, 155411 (2012).

48 D.F. Urban, R. Avriller, and A. Levy Yeyati, Phys. Rev. B 82, 121414 (2010); T. Novotný, F. Haupt, and W. Belzig, Phys. Rev. B 84, 113107 (2011)

49 T.H. Park and M. Galperin, Phys. Rev. B 84, 205450 (2011).

50 Y. Utsumi, O. Entin-Wohlman, A. Ueda, and A. Aharony, Phys. Rev. B 87, 115407 (2013).

51 S. Maier, T.L. Schmidt, and A. Komnik, Phys. Rev. B 83, 085401 (2011).

52 G.D. Mahan, Many-Particle Physics. (Third edition, Kluwer Academic/Plenum Publisher, New York, 2000).

53 M.K. Grover and R. Silbey, J. Chem. Phys. 52, 2099 (1970).

54 J. Tobiska and Yu.V. Nazarov, Phys. Rev. B 72, 235328 (2005).

55 H. Förster and M. Büttiker, Phys. Rev. Lett. 101, 136805 (2008).

56 S. Engelsberg and J.R. Schrieffer, Phys. Rev. 131, 993 (1963). 